\title{
A feasible, effective image transmitting framework in WMSNs
}

\author{
Feng Tian ${ }^{1,}$ a,$K_{\text {Kun Song }}^{1, b^{*}}$ and Enyan Sun ${ }^{1, c}$ \\ ${ }^{1}$ School of Computer Science, Shenyang Aerospace University, Shenyang 110136, China \\ atianfeng5861@163.com, bsongkun2008@gmail.com, csunenyan418418@163.com
}

Keywords: WMSNs, ROI, distributed DWT calculation, SPIHT, the life time of network

Abstract. In WMSNs, compared with the complicated media processing task the energy of node is very limited. In this paper, we use ROI coding of the image to decrease the requirement of the energy, and propose a feasible, effective framework to prolong the life time of the network.

\section{Introduction}

The wireless multimedia sensor networks now are widely applied in many kinds of domains, and the critical problem between the constrained energy of node and the highly demanded calculation resource is focused by many researchers. In [1], the author solves this problem by distributing the DWT calculation to different clusters to homogenize the cost of the energy to prolong the life time of the network. In [2], the author distributes the DCT calculation to different clusters to homogenize the cost of the energy. In [3], the author solves this problem by using information theory to maximize the overall compression of the visual information from the view of the spatial correlation. In [4], the author solves the problem by using image fusing technology to eliminate the redundant information during the transmission.

The papers mentioned above all propose the solving method from their own perspective separately. Furthermore, in this paper, we introduce the ROI(Region of Interest)[5] coding of the image and propose a feasible, effective framework to solve the same problem in WMSNs. In the framework we propose, the heavy calculation power is mainly put into the host terminal.

The rest of this paper is organized as follows: In section 2, we firstly illustrate the technology we use in the framework. In section 3, we illustrate the framework we design. In section 4, we show the simulation, calculation and the result of performance evaluation. In section 5, we conclude this paper and give the future work.

\section{The relative technology}

The Background of Image Compression Using DWTs

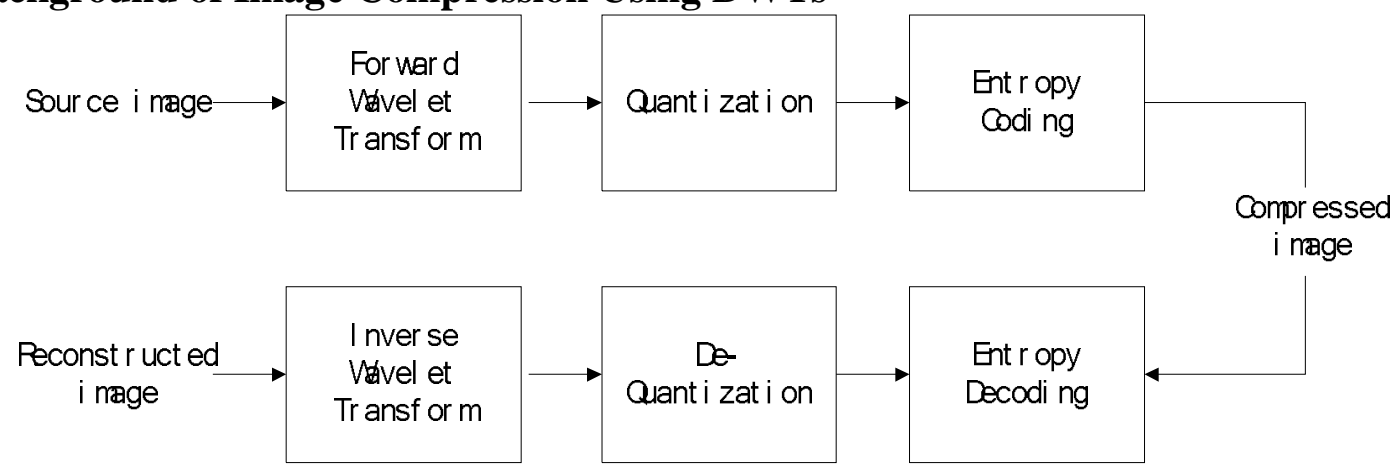

Fig. 1.The process of the image compression using DWT

As is shown in fig.1, the original image is firstly transformed by the discrete wavelet transform, then the wavelet matrix is quantified by using compressing algorithm (e.g., JPEG2000, SPIHT[6],etc.), at last the bit stream is still needed to be entropy coded to furthermore decrease the volume of bit stream. In this paper, we use the SPIHT algorithm to do the quantization. 
The Framework of ROI Image Coding Based on SPIHT

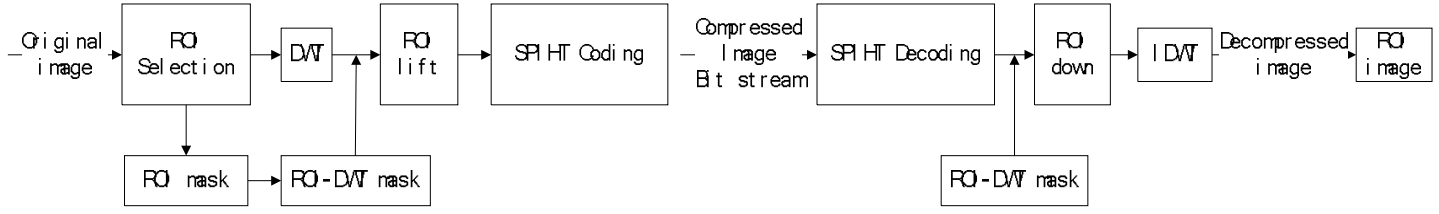

Frantework of $\mathrm{RO}$ i nage coding based on SP HT

Fig. 2.The framework of ROI image coding based on SPIHT

The framework of ROI image coding based on SPIHT which is used in this paper is shown in fig. 2. It is noted that we omit the entropy coding procedure in order to simply the problem description.

The two important points of this algorithm framework are the ROI-DWT mask, which refers to the ROI mask information in the small wavelet domain, and the ROI lift which refers to the lifting of the bit plane of ROI aiming to ensure that the ROI is encoded earlier than BG. The ROI-DWT mask can be generated as follows: firstly, ROI is selected in the original image to acquire the ROI mask, and then the ROI mask is transformed into the ROI-DWT mask. The fig. 3(a), fig. 3(b), and fig. 3(c) respectively show the original image, the ROI mask and the ROI-DWT mask. The method of transforming of the ROI-DWT mask includes: we imagine that the fig. $3 \mathrm{~b}$ is seen as the zero level small wavelet sub-band, for the $n$ level small wavelet transformation, when the level I varies from 1 to $\mathrm{n}$, every coefficient of the I level small wavelet checks whether any of the four nodes in the i- 1 level sub-band is ROI coefficient (for example, 1). In other words, if any of the four nodes is 1 , then this node will be assigned 1 , otherwise 0 . Finally, we will get a binary tree, as the equation (1) defines:

$$
R O_{D W}(i, j)=\left\{\begin{array}{lll}
1, & \text { if } & \operatorname{Dest}(i, j)=1 \\
0, & \text { if } & \operatorname{Dest}(i, j)=0
\end{array}\right. \text {. }
$$

For every node in this tree, if every of its descendant nodes is 1 , then this node is 1 , otherwise 0 . This tree is the ROI mask of the small wavelet domain, which we define as ROI-DWT mask. The ROI lift means multiplying the small wavelet coefficients which are in correspondence with the ROI-DWT mask with $2^{s}$, and it will assure the SPIHT algorithm firstly encode the ROI.

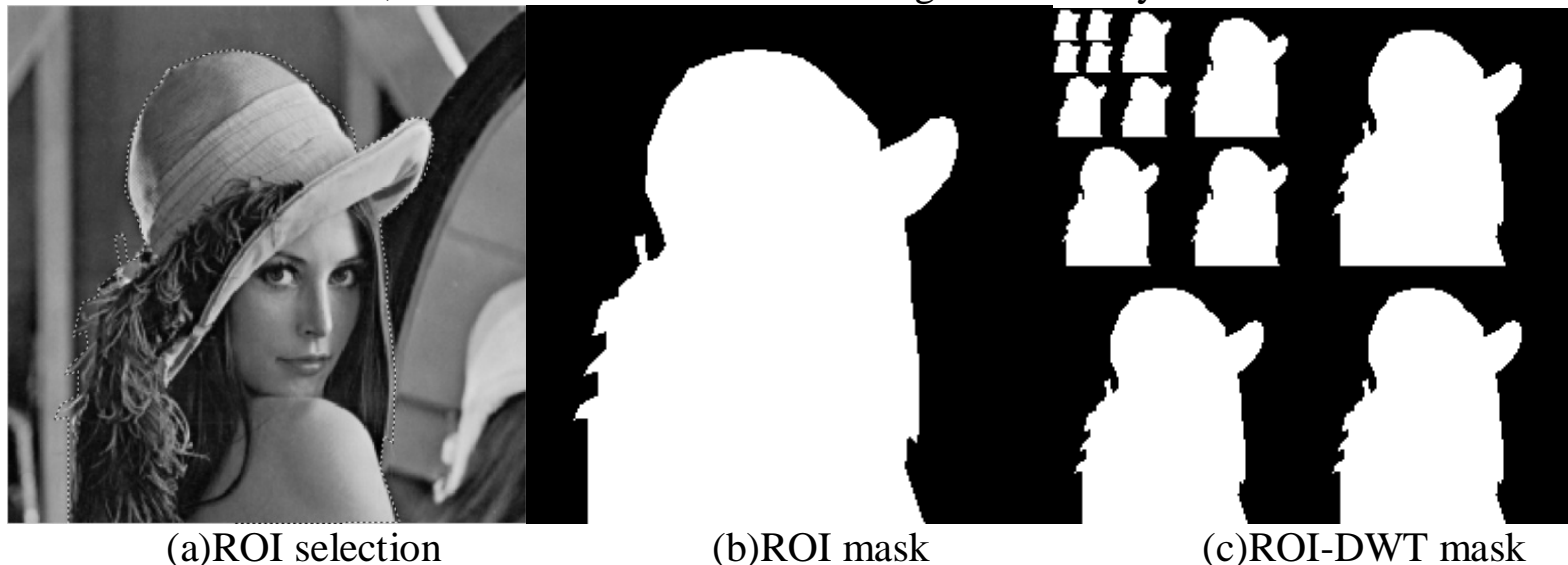

Fig. 3. The generating process of ROI-DWT mask

In this paper, by calculating a $2^{S}$ which is big enough, we can assure the whole ROI is encoded in higher priority than BG (Back Ground). Moreover we can only encode the ROI, and don't encode BG. By doing this, we can save the energy. This is like the method called MAXSHIFT method in JPEG2000[6], but according to the topology of the WMSNs in fig. 4,we know the contour information of the ROI, and we can put the complicated calculation in the host terminal rather than sensor nodes, so the energy of the WMSNs can be saved as a total result. 


\section{The framework we proposed}

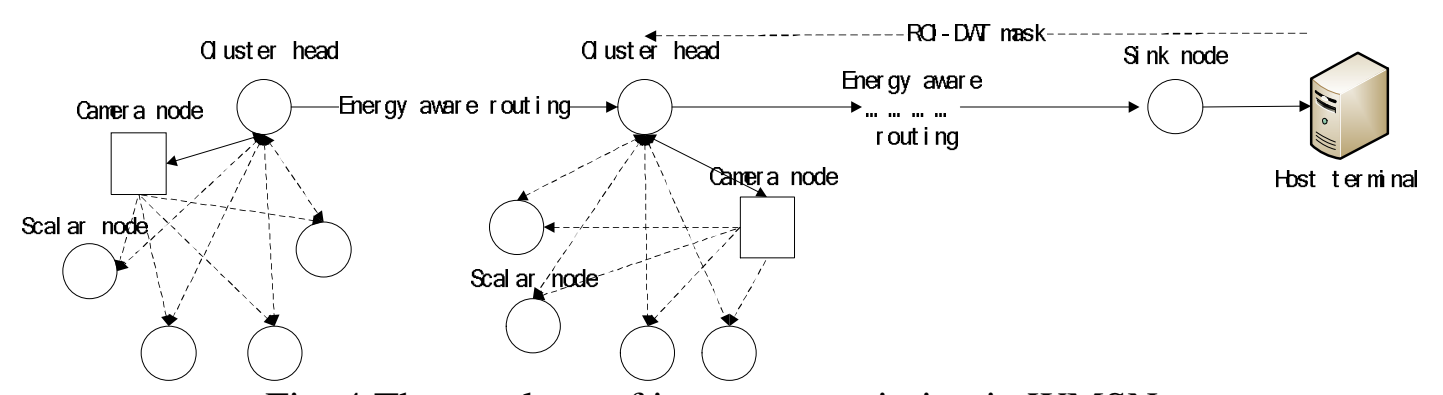

Fig. 4.The topology of image transmission in WMSNs

As is shown in fig. 4 compared with fig. 5 below, mass calculation can be completed in host terminal, and the sensor nodes only need to execute the DWT, ROI lift and SPIHT Coding procedure. We design this kind of coding and transmitting framework to reduce the energy cost of WMSNs. Furthermore, in every cluster, we apply distributed DWT calculation to homogenize the energy cost of every node, and introduce energy aware routing[7] to homogenize the energy cost of the cluster header nodes when the image is transmitted to the host terminal, so by using the methods above we prolong the lifetime of the network.

The whole framework of image transmission can be concluded as follows:

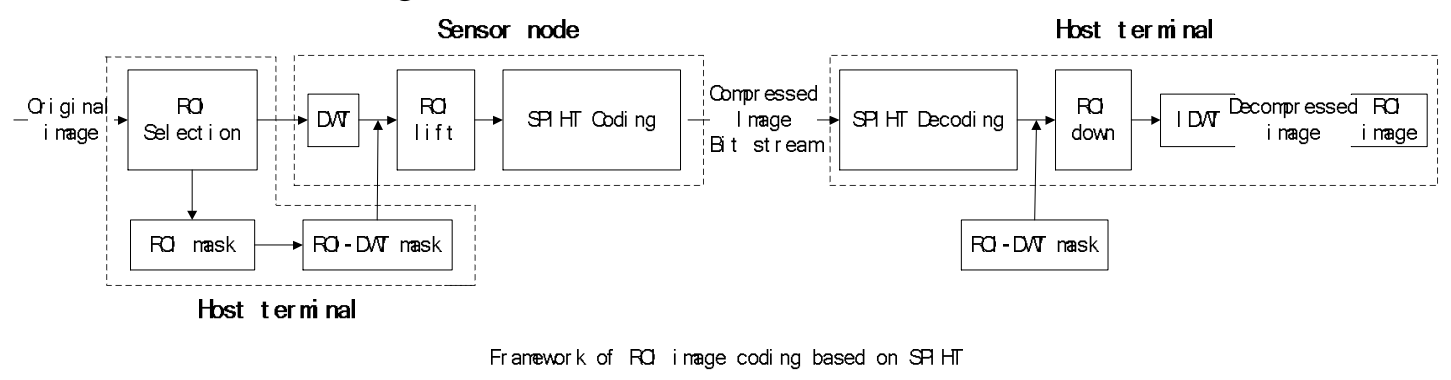

Fig. 5.The framework of ROI image coding based on SPIHT

We assume that there is one camera node and some scalar nodes in one cluster, and the cluster header node is the node whose residual energy is the largest. This situation can be easily extended to the situation that multi camera nodes exist in one cluster.

1) The camera node takes the image, and asks the cluster header node how many scalar nodes are there in its cluster; the cluster header node replies the number $\mathrm{n}$ of the scalar nodes to it. Then the camera node divides its image to $\mathrm{n}$ tiles and transmits them to every scalar node. The scalar nodes execute one level small wavelet decomposition and transmit their decomposition result to the cluster header node[1,8]. The cluster header node divides LL sub-band of current level to $\mathrm{n}$ parts and transmits them to the scalar nodes to execute next level decomposition. This process continues until it reaches the required decomposition level. This process is shown in fig. 4;

2) After the decomposition, the cluster header node compresses the wavelet coefficients using SPIHT algorithm and transmits the image to sink node by energy aware routing, and then the sink node transmits the image to host terminal;

3) In host terminal, there is the most numerous calculations which can occur. User may select the monitor area as the ROI, or select the special area which is designated by the image stitching technology as the ROI for the overlapped images, and then calculate the ROI mask and the ROI-DWT mask, and transmit the ROI-DWT mask back to the cluster header node;

4) The camera node executes the procedure same to 1 );

5) This procedure is similar to 2), but at this time the cluster header node executes the ROI-lift and only need to encode the ROI and transmit it, which is aforementioned in section 2.2;

6) In host terminal, user can observe the monitor area, or recover the image whose Field of View is larger by using the image stitching technology.

In the next time when the camera node takes the image, the procedure will always begin from 4).In this framework, the energy aware routing refers to the route which can homogenize the energy cost of the cluster headers. 


\section{Performance and evaluation}

To verify the effect of the framework, we use matlab7.0 to simulate the ROI coding in higher priority, and use energy model to observe the energy cost of one cluster.

The example image is lena $256 \times 256$ pixels with 8 bits per pixel. The wavelet filter is bior 4.4 , and the decomposition level is set to 4 .

To test the effect of encoding the ROI in higher priority, the $2^{S}$ is needed to be 67108864 which is big enough to ensure the minimum coefficient in ROI is bigger than the maximum coefficient in BG through calculation. The test result of the encoding length, encoding time, PSNR and display effect is as follows:

Table.1.The test result of different quantization parts

\begin{tabular}{|c|c|c|c|c|}
\hline $\begin{array}{c}\text { Quantization } \\
\text { Object }\end{array}$ & $\begin{array}{c}\text { Length } \\
\text { (bits) }\end{array}$ & $\begin{array}{c}\text { Encoding } \\
\text { Time } \\
\text { (seconds) }\end{array}$ & $\begin{array}{c}\text { PSNR } \\
\text { (decibels) }\end{array}$ & Display Effect \\
\hline $\begin{array}{c}\text { ROI in } \\
\text { Figure3(a) }\end{array}$ & 32659 & 1.484 & 10.79 & \\
\hline & & & & \\
Whole image & 65536 & 3.512 & 10.81 & \\
\hline
\end{tabular}

From the table 1, we can see that by only encoding the ROI, the time of encoding will decrease correspondingly, and the display effect of the images has no much difference.

To check the energy cost of every node in one cluster, we use a transceiver energy dissipation model same to the model in [1] here. The energy consumed in transmission per bit is

$$
E_{T X}=\varepsilon_{e}+\varepsilon_{a} d^{\alpha}
$$

and the energy consumed in reception per bit is

$$
E_{R X}=\varepsilon_{e}
$$


Where $\varepsilon_{a}$ is the energy dissipated per bit per $m^{2}, \varepsilon_{e}$ is energy consumed by the circuit per bit, $\mathrm{d}$ is the distance between a wireless transmitter and a receiver, and $2 \leq \alpha \leq 4$ is the path loss parameter. The energy consumed in computation per bit is

$$
E_{D W}=\gamma
$$

Where $\gamma$ is the energy dissipated for one level wavelet transform compression per bit. The energy spent in quantization and entropy coding per bit is

$$
E_{Q N T}=\delta
$$

The values of the parameters of the wireless communication energy model (2) and (3) are typical values $\varepsilon_{a}=100 \times 10^{-12}$ Joul e / bi $t / \mathrm{m}^{2}$ and $\varepsilon_{e}=50 \times 10^{-9} \mathrm{Joul} e / \mathrm{bi} t$, the communication range of a node $d$ is chosen to be $10 \mathrm{~m}$ for all nodes, and $\alpha=2$. The value of $\gamma$ in (4) is estimated in [1] to be $220 \times 10^{-9} \mathrm{Joul} e /$ bi $t$ and the value of $\delta$ in (5) is estimated in [1] to be $20 \times 10^{-9}$ Joul e / bi $t$. The size of the image is represented by Size, which is equal to $256 * 256 * 8$ bits. The compression ratio is represented by Ratio, and when the bit per pixel is set to 1.0, the Ratio can achieve $12.30 \%$ in our paper. The ROI coding part divided by the complete bit stream is equal to 1 when there is no ROI, or is equal to $0.67(32659 / 65536)$ in the test result in table1, and the quotient is represented by RR. We imagine that there are $\mathrm{n}$ sensor nodes in one cluster.

The calculation is as follows:

When we don't use the distributed transformation algorithm which is depicted in Section 2:

The energy cost of Camera node:

$S i z e \times E_{D W} \times\left(1+\frac{1}{4}+\frac{1}{16}+\frac{1}{64}\right)+S i z e \times E_{B V T} \times R R+S i z e \times R a t i o \times R R \times E_{T X}=S z e \times$

$\left.220 \times\left(1+\frac{1}{4}+\frac{1}{16}+\frac{1}{64}\right)+S z e \times 20 \times 0.67+S z e \times 12.3 \% \times 0.67 \times 60 \approx S z e \times 310.536\right)$

The energy cost of the Cluster header node:

$S i z e \times R R \times$ Ratio $\times\left(E_{R X}+E_{T X}\right)=S i z e \times 0.67 \times 12.3 \% \times(50+60) \approx S i z e \times 9.0651(7)$

When we use the distributed transformation algorithm:

The energy cost of Camera node:

$S i z \times E_{T X}=S i z e \times 60$

The energy cost of Sensor node:

$$
\begin{aligned}
& S z e \times \frac{1}{n} \times\left(E_{R X}+E_{D W T}+E_{T X}\right) \times\left(1+\frac{1}{4}+\frac{1}{16}+\frac{1}{64}\right)=S i z \times \frac{1}{n} \times(50+220+60) \times\left(1+\frac{1}{4}\right. \\
& \left.+\frac{1}{16}+\frac{1}{64}\right) \approx S i z e \times \frac{1}{n} \times 438.28
\end{aligned}
$$


The energy cost of the Cluster header node:

$$
\begin{aligned}
& S z e \times E_{R X}+S z e \times\left(E_{T X}+E_{R X}\right) \times\left(\frac{1}{4}+\frac{1}{16}+\frac{1}{64}\right)+S z e \times R R \times E_{B N T}+S z e \times R R \times \\
& \text { Rat i } 0 \times E_{T X}=S i z e \times 50+S i z e \times(50+60) \times\left(\frac{1}{4}+\frac{1}{16}+\frac{1}{64}\right)+S z e \times 0.67 \times 20+ \\
& \text { S } z e \times 0.67 \times 12.3 \% \times 60 \approx S i z e \times 104.44
\end{aligned}
$$

From the calculation above, we can see the energy cost of the camera node will be greatly reduced. The energy cost of the cluster header node is a constant, approximately $S z e \times 104$. 44. When $n$ is equal to 4, the energy cost of the sensor node is nearly equal to the energy cost of the cluster header node, and it means that every time when the image is taken there is no need to reelect the cluster header node which should always have the largest residual energy in our imagination. With the growth of $n$, the life time of the network will increase correspondingly.

\section{Conclusion and future work}

In this paper, we study the problem how to prolong the life time of the WMSNs, and propose a kind of new framework which emphasizes specially the image ROI coding and distributed DWT calculation. In the future, we may put the technology into the testbed, and there are still many relative research needed to do, such as the extension of energy-aware routing and the combination of the image stitching technology.

\section{References}

[1] H.Wu, andAlhussein A. Abouzeid: "Energy Efficient Distributed Image Compression in Resource Constrained Multihop Wireless Networks", Computer Communications, 2005, 28, 14, (1), pp.1658-1668

[2] Xiaobo Yang, Lijuan Sun, Ruchuan Wang: "Distributed Image Compression Algorithm in Wireless Multimedia Sensor Networks”, ZTE COMMUNICATIONS, 2010, 8, (1), pp.50-54

[3] P. Wang, R. Dai, I.F.Akyildiz: "A Spatial Correlatio-Based Image Compression FrameWork for Wireless Multimedia Sensor Networks”, IEEE Trans. Multimedia, 2011, 13, (2), pp.388-401

[4] Rong Wu: " Research on Data Fusion Technology of Wireless Multimedia Sensor Networks ". Master's thesis, Nangjing University of Posts and Telecommunications, 2012

[5] C.A.Christopoulos,T.Ebrahimi, A.N.Skodras: "JPEG2000: the new still picture compression standard". Proceedings of the 2000 ACM workshops on Multimedia. Los Angels, California, United States, 2000, pp .45-49

[6] A.Said and W.A.Pearlman: "A new fast/efficient image codec based on set partitioning in hierarchical trees”, IEEE trans.CircuitsSyst.Video Technol., 1996, 6, (12), pp. 243-250

[7] Shah,R.C., Rabaey,J.M.: "Energy Aware Routing for Low Energy Ad Hoc Sensor Networks". Wireless Communications and Networking Conference,2002.WCNC2002. 2002 IEEE , Orlando, USA, 2002, 1, pp. 350-355

[8] G. Sadashivappa,MaheshJayakar,K.V.S.AnandaBabu: "Analysis of SPIHT Algorithm Using Tiling Operations". Signal Acquisition and Processing, 2010. ICSAP 10. International Conference, Bangalore, India, 2010, pp. 332-336 